\title{
Realization of Perfect Reconstruction Non-uniform Filter Banks via a Tree Structure
}

\author{
Wing-kuen Ling and P. K. S. Tam \\ Department of Electronic and Information Engineering \\ The Hong Kong Polytechnic University \\ Hung Hom, Kowloon, Hong Kong \\ Hong Kong Special Administrative Region, China \\ Tel: (852) 2766-6238, Fax: (852) 2362-8439 \\ bingodencserver.eie.polyu.edu.hk
}

\begin{abstract}
Obviously, a tree structure filter bank can be realized via a nonuniform filter bank, and perfect reconstruction is achieved if and only if each branch of the tree structure can provide perfect reconstruction. In this paper, the converse of this problem is studied. We show that a perfect reconstruction non-uniform filter bank with decimation ratio $\{2,4,4\}$ can be realized via a tree structure and each branch of the tree structure achieves perfect reconstruction.
\end{abstract}

\section{Introduction}

It is well known that the tree structure filter bank shown in figure $1 \mathrm{~b}$ can be realized via a non-uniform filter bank shown in figure 1a, and perfect reconstruction can be achieved if and only if each branch of the tree structure can provide perfect reconstruction [1-4]. However, is the converse true? That is, given any perfect reconstruction non-uniform filter shown in figure 1a, can it be realized via a tree structure shown in figure $1 \mathrm{~b}$ ? In general, a perfect reconstruction non-uniform filter bank cannot be realized by a tree structure [11]. This paper works on this problem.

There are some advantages of realizing a non-uniform filter bank via a tree structure, such as reducing the filter length in the filters [5], and improving the computation complexity and implementation speed [5]. In section 2, we show how a perfect reconstruction non-uniform filter bank can be converted to a tree structure filter bank. Some illustrative examples are demonstrated in section 3. Finally, a conclusion is given in section 4 .

\section{Realization of Non-uniform Filter Bank Via a Tree Structure}

\section{Theorem 1}

A non-uniform filter bank with decimation ratio $\{2,4,4\}$ achieves perfect reconstruction if and only if it can be realized via a tree structure and each branch of the tree structure achieves perfect reconstruction. 
Proof:

Since the if part was well known [1-4], we only prove the only if part.

A non-uniform filter bank shown in figure 1a achieves perfect reconstruction if and only if $\exists c \in \mathbf{C}$ and $\exists m \in \mathbf{Z}$ such that:

$$
\left(\left[\begin{array}{ccc}
\frac{1}{2} \cdot H_{0}(z) & \frac{1}{4} \cdot H_{1}(z) & \frac{1}{4} \cdot H_{2}(z) \\
0 & \frac{1}{4} \cdot H_{1}(z \cdot W) & \frac{1}{4} \cdot H_{2}(z \cdot W) \\
\frac{1}{2} \cdot H_{0}\left(z \cdot W^{2}\right) & \frac{1}{4} \cdot H_{1}\left(z \cdot W^{2}\right) & \frac{1}{4} \cdot H_{2}\left(z \cdot W^{2}\right) \\
0 & \frac{1}{4} \cdot H_{1}\left(z \cdot W^{3}\right) & \frac{1}{4} \cdot H_{2}\left(z \cdot W^{3}\right)
\end{array}\right]\right) \cdot\left(\begin{array}{c}
G_{0}(z) \\
G_{1}(z) \\
G_{2}(z)
\end{array}\right)=\left(\begin{array}{c}
c \cdot z^{m} \\
0 \\
0 \\
0
\end{array}\right) \cdot
$$

This directly implies that:

$$
\operatorname{det}\left(\left[\begin{array}{cc}
H_{1}(z \cdot W) & H_{2}(z \cdot W) \\
H_{1}\left(z \cdot W^{3}\right) & H_{2}\left(z \cdot W^{3}\right)
\end{array}\right]\right)=0 .
$$

Since

$\operatorname{det}\left(\left[\begin{array}{cc}H_{1}(z \cdot W) & H_{2}(z \cdot W) \\ H_{1}\left(z \cdot W^{3}\right) & H_{2}\left(z \cdot W^{3}\right)\end{array}\right]\right)=0 \Rightarrow \operatorname{det}\left(\left[\begin{array}{cc}H_{1}(z) & H_{2}(z) \\ H_{1}\left(z \cdot W^{2}\right) & H_{2}\left(z \cdot W^{2}\right)\end{array}\right]\right)=0$,

hence

$$
\begin{aligned}
& \left(\left[\begin{array}{ccc}
\frac{1}{2} \cdot H_{0}(z) & \frac{1}{4} \cdot H_{1}(z) & \frac{1}{4} \cdot H_{2}(z) \\
0 & \frac{1}{4} \cdot H_{1}(z \cdot W) & \frac{1}{4} \cdot H_{2}(z \cdot W) \\
\frac{1}{2} \cdot H_{0}\left(z \cdot W^{2}\right) & \frac{1}{4} \cdot H_{1}\left(z \cdot W^{2}\right) & \frac{1}{4} \cdot H_{2}\left(z \cdot W^{2}\right) \\
0 & \frac{1}{4} \cdot H_{1}\left(z \cdot W^{3}\right) & \frac{1}{4} \cdot H_{2}\left(z \cdot W^{3}\right)
\end{array}\right]\right) \cdot\left(\begin{array}{c}
G_{0}(z) \\
G_{1}(z) \\
G_{2}(z)
\end{array}\right)=\left(\begin{array}{c}
c \cdot z^{m} \\
0 \\
0 \\
0
\end{array}\right) \\
& \Rightarrow \operatorname{det}\left(\left[\begin{array}{cc}
H_{1}(z) & H_{2}(z) \\
H_{1}(z \cdot W) & H_{2}(z \cdot W)
\end{array}\right]\right) \neq 0
\end{aligned}
$$

and

$$
\operatorname{det}\left(\left[\begin{array}{cc}
H_{0}(z) & H_{2}(z) \\
H_{0}\left(z \cdot W^{2}\right) & H_{2}\left(z \cdot W^{2}\right)
\end{array}\right]\right) \neq 0
$$


The converse is also true. That is if:

$$
\begin{gathered}
\operatorname{det}\left(\left[\begin{array}{cc}
H_{1}(z) & H_{2}(z) \\
H_{1}(z \cdot W) & H_{2}(z \cdot W)
\end{array}\right]\right) \neq 0, \\
\operatorname{det}\left(\left[\begin{array}{cc}
H_{0}(z) & H_{2}(z) \\
H_{0}\left(z \cdot W^{2}\right) & H_{2}\left(z \cdot W^{2}\right)
\end{array}\right]\right) \neq 0
\end{gathered}
$$

and

$$
\operatorname{det}\left(\left[\begin{array}{cc}
H_{1}(z \cdot W) & H_{2}(z \cdot W) \\
H_{1}\left(z \cdot W^{3}\right) & H_{2}\left(z \cdot W^{3}\right)
\end{array}\right]\right)=0,
$$

then there exist $G_{0}(z), G_{l}(z)$ and $G_{2}(z)$ such that:

$$
\left(\left[\begin{array}{ccc}
\frac{1}{2} \cdot H_{0}(z) & \frac{1}{4} \cdot H_{1}(z) & \frac{1}{4} \cdot H_{2}(z) \\
0 & \frac{1}{4} \cdot H_{1}(z \cdot W) & \frac{1}{4} \cdot H_{2}(z \cdot W) \\
\frac{1}{2} \cdot H_{0}\left(z \cdot W^{2}\right) & \frac{1}{4} \cdot H_{1}\left(z \cdot W^{2}\right) & \frac{1}{4} \cdot H_{2}\left(z \cdot W^{2}\right) \\
0 & \frac{1}{4} \cdot H_{1}\left(z \cdot W^{3}\right) & \frac{1}{4} \cdot H_{2}\left(z \cdot W^{3}\right)
\end{array}\right]\right) \cdot\left(\begin{array}{c}
G_{0}(z) \\
G_{1}(z) \\
G_{2}(z)
\end{array}\right)=\left(\begin{array}{c}
c \cdot z^{m} \\
0 \\
0 \\
0
\end{array}\right) .
$$

Let

$$
H_{i}(z)=\sum_{l=0}^{3} z^{-l} \cdot E_{i, l}\left(z^{4}\right),
$$

for $i=0,1,2$, then from equation (2), there exist $R(z), R^{\prime}(z)$ and $R^{\prime \prime}(z)$ such that:

$$
\begin{gathered}
E_{1,1}\left(z^{4}\right)=R\left(z^{4}\right) \cdot E_{1,0}\left(z^{4}\right) \text { and } E_{2,1}\left(z^{4}\right)=R\left(z^{4}\right) \cdot E_{2,0}\left(z^{4}\right) \text { and } \\
E_{1,3}\left(z^{4}\right)=R^{\prime}\left(z^{4}\right) \cdot E_{1,2}\left(z^{4}\right) \text { and } E_{2,3}\left(z^{4}\right)=R^{\prime}\left(z^{4}\right) \cdot E_{2,2}\left(z^{4}\right) \text { and } \\
\left\{R\left(z^{4}\right)=R^{\prime}\left(z^{4}\right) \text { or }\left\{E_{1,0}\left(z^{4}\right)=R^{\prime \prime}\left(z^{4}\right) \cdot E_{1,2}\left(z^{4}\right) \text { and } E_{2,0}\left(z^{4}\right)=R^{\prime \prime}\left(z^{4}\right) \cdot E_{2,2}\left(z^{4}\right)\right\}\right\} .
\end{gathered}
$$

But $E_{1,0}\left(z^{4}\right)=R ”\left(z^{4}\right) \cdot E_{1,2}\left(z^{4}\right)$ and $E_{2,0}\left(z^{4}\right)=R ”\left(z^{4}\right) \cdot E_{2,2}\left(z^{4}\right)$ contradict equation (4). Hence, we have $R\left(z^{4}\right)=R^{\prime}\left(z^{4}\right) . R\left(z^{4}\right)=R^{\prime}\left(z^{4}\right)$, which implies:

$$
\frac{H_{2}(z)}{H_{1}(z)}=\frac{E_{2,0}\left(z^{4}\right)+z^{-2} \cdot E_{2,2}\left(z^{4}\right)}{E_{1,0}\left(z^{4}\right)+z^{-2} \cdot E_{1,2}\left(z^{4}\right)}
$$


Hence, there exist $F^{\prime}{ }_{l}(z), F_{0}(z)$, and $F_{l}(z)$ such that $H_{l}(z)=F^{\prime}{ }_{l}(z) \cdot F_{0}\left(z^{2}\right)$ and $H_{2}(z)=F^{\prime}{ }_{l}(z) \cdot F_{l}\left(z^{2}\right)$, respectively. And the non-uniform filter bank shown in figure 1a can be realized via a tree structure shown in figure $1 \mathrm{~b}$.

From equation (4), we have:

$$
\begin{gathered}
E_{1,2}\left(z^{4}\right) \cdot E_{2,0}\left(z^{4}\right)-E_{1,0}\left(z^{4}\right) \cdot E_{2,2}\left(z^{4}\right) \neq 0 \text { and } \\
\left\{E_{0,1}\left(z^{4}\right) \cdot E_{2,0}\left(z^{4}\right)-E_{0,0}\left(z^{4}\right) \cdot E_{2,1}\left(z^{4}\right) \neq 0\right. \text { or } \\
E_{0,3}\left(z^{4}\right) \cdot E_{2,2}\left(z^{4}\right)-E_{0,2}\left(z^{4}\right) \cdot E_{2,3}\left(z^{4}\right) \neq 0 \text { or } \\
\left.E_{0,1}\left(z^{4}\right) \cdot E_{2,2}\left(z^{4}\right)+E_{0,3}\left(z^{4}\right) \cdot E_{2,0}\left(z^{4}\right)-E_{0,0}\left(z^{4}\right) \cdot E_{2,3}\left(z^{4}\right)-E_{0,2}\left(z^{4}\right) \cdot E_{2,1}\left(z^{4}\right) \neq 0\right\} .
\end{gathered}
$$

Let $F_{l}\left(z^{2}\right)$ be the numerator of $H_{2}(z) / H_{l}(z)$, and $F_{0}\left(z^{2}\right)$ be the denominator of $H_{2}(z) / H_{l}(z)$, respectively. We have:

$\operatorname{det}\left(\left[\begin{array}{cc}F_{0}(z) & F_{1}(z) \\ F_{0}(-z) & F_{1}(-z)\end{array}\right]\right)=2 \cdot z^{-1} \cdot\left(E_{1,2}\left(z^{2}\right) \cdot E_{2,0}\left(z^{2}\right)-E_{1,0}\left(z^{2}\right) \cdot E_{2,2}\left(z^{2}\right)\right) \neq 0$,

and

$$
\begin{aligned}
\operatorname{det}\left(\left[\begin{array}{cc}
H_{0}(z) & F_{1}^{\prime}(z) \\
H_{0}(-z) & F_{1}^{\prime}(-z)
\end{array}\right]\right)= & \frac{2 \cdot z^{-1} \cdot\left(E_{0,1}\left(z^{4}\right) \cdot E_{2,0}\left(z^{4}\right)-E_{0,0}\left(z^{4}\right) \cdot E_{2,1}\left(z^{4}\right)\right)+2 \cdot z^{-5} \cdot\left(E_{0,3}\left(z^{4}\right) \cdot E_{2,2}\left(z^{4}\right)-E_{0,2}\left(z^{4}\right) \cdot E_{2,3}\left(z^{4}\right)\right)}{E_{2,0}\left(z^{4}\right)+z^{-2} \cdot E_{2,2}\left(z^{4}\right)} \\
& +\frac{2 \cdot z^{-3} \cdot\left(E_{0,1}\left(z^{4}\right) \cdot E_{2,2}\left(z^{4}\right)+E_{0,3}\left(z^{4}\right) \cdot E_{2,0}\left(z^{4}\right)-E_{0,0}\left(z^{4}\right) \cdot E_{2,3}\left(z^{4}\right)-E_{0,2}\left(z^{4}\right) \cdot E_{2,1}\left(z^{4}\right)\right)}{E_{2,0}\left(z^{4}\right)+z^{-2} \cdot E_{2,2}\left(z^{4}\right)} \\
& \neq 0 .
\end{aligned}
$$

Hence, each branch of the tree structure achieves perfect reconstruction.

\section{Illustrative Examples}

\subsection{Non-tree Structure Filter Bank}

Consider an example of $H_{0}(z)=1+z^{-1}, H_{1}(z)=1-z^{-1}$, and $H_{2}(z)=z^{-2}$, respectively. Since $H_{1}(z) / H_{2}(z)=z^{2} \cdot\left(1-z^{-1}\right)$, there does not exist $F^{\prime}{ }_{1}(z), F_{0}(z)$, and $F_{1}(z)$ such that $H_{l}(z)=F^{\prime}{ }_{l}(z) \cdot F_{0}\left(z^{2}\right)$ and $H_{2}(z)=F^{\prime}{ }_{l}(z) \cdot F_{l}\left(z^{2}\right)$, respectively. Hence, this non-uniform filter bank cannot be realized via a tree structure. By theorem 1, this non-uniform filter bank does not achieve perfect reconstruction.

It is worth to note that by converting the non-uniform filter bank to a uniform filter bank shown in figure 2 [6-10], perfect reconstruction can be achieved. However, $G^{\prime}{ }_{-1}(z) \neq z^{-2} \cdot G^{\prime}{ }_{0}(z)$, this implies that the corresponding synthesis filter $G_{0}(z)$ shown in figure $1 \mathrm{a}$ is time varying. 


\subsection{Tree Structure Filter Bank}

Consider another example with $H_{0}(z)=2 \cdot\left(1+z^{-1}+z^{-2}+z^{-3}\right), H_{1}(z)=4 \cdot\left(2+6 \cdot z^{-1}+4 \cdot z^{-}\right.$ $\left.{ }^{2}+12 \cdot z^{-3}\right)$, and $H_{2}(z)=4 \cdot\left(5+15 \cdot z^{-1}+7 \cdot z^{-2}+21 \cdot z^{-3}\right)$, respectively. Since $H_{1}(z) / H_{2}(z)=\left(2+4 \cdot z^{-2}\right) /\left(5+7 \cdot z^{-2}\right)$, there exists $F^{\prime}{ }_{1}(z), F_{0}(z)$, and $F_{l}(z)$ such that $H_{1}(z)=F^{\prime}{ }_{1}(z) \cdot F_{0}\left(z^{2}\right)$ and $H_{2}(z)=F^{\prime}{ }_{1}(z) \cdot F_{1}\left(z^{2}\right)$, respectively. Hence, this non-uniform filter bank can be realized via a tree structure. It can be checked easily that each branch in the tree structure achieves perfect reconstruction. Hence, this non-uniform filter bank achieves perfect reconstruction.

\section{Conclusion}

In this paper, we show that a non-uniform filter bank with decimation ratio $\{2,4,4\}$ achieves perfect reconstruction if and only if it can be realized via a tree structure and each branch of the tree structure achieves perfect reconstruction. The advantage of realizing a non-uniform filter bank via a tree structure is to reduce the computation complexity and provide a fast implementation for a non-uniform filter bank [5].

\section{Acknowledgement}

The work described in this paper was substantially supported by a grant from the Hong Kong Polytechnic University with account number G-V968.

\section{References}

1. Vaidyanathan P. P.: Lossless Systems in Wavelet Transforms. IEEE International Symposium on Circuits and Systems, ISCAS, Vol. 1. (1991) 116-119.

2. Soman K. and Vaidyanathan P. P.: Paraunitary Filter Banks and Wavelet Packets. IEEE International Conference on Acoustics, Speech, and Signal Processing, ICASSP, Vol. 4. (1992) 397-400.

3. Sodagar I., Nayebi K. and Barnwell T. P.: A Class of Time-Varying Wavelet Transforms. IEEE International Conference on Acoustics, Speech, and Signal Processing, ICASSP, Vol. 3. (1993) 201-204.

4. Soman A. K. and Vaidyanathan P. P.: On Orthonormal Wavelets and Paraunitary Filter Banks. IEEE Transactions on Signal Processing, Vol. 41, No. 3. (1993) 1170-1183.

5. Vaidyanathan P. P.: Multirate Systems and Filter Banks. Englewood Cliffs, NJ: Prentice Hall, 1993.

6. Hoang P. Q. and Vaidyanathan P. P.: Non-Uniform Multirate Filter Banks: Theory and Design. IEEE International Symposium on Circuits and Systems, ISCAS, Vol. 1. (1989) 371-374. 
7. Li J., Nguyen T. Q. and Tantaratana S.: A Simple Design Method for Nonuniform Multirate Filter Banks. Conference Record of the Twenty-Eight Asilomar Conference on Signals, Systems and Computers, Vol. 2. (1995) 10151019.

8. Makur A.: BOT's Based on Nonuniform Filter Banks. IEEE Transactions on Signal Processing, Vol. 44, No. 8. (1996) 1971-1981.

9. Li J., Nguyen T. Q. and Tantaratana S.: A Simple Design Method for NearPerfect-Reconstruction Nonuniform Filter Banks. IEEE Transactions on Signal Processing, Vol. 45, No. 8. (1997) 2105-2109.

10. Omiya N., Nagai T., Ikehara M. and Takahashi S. I.: Organization of Optimal Nonuniform Lapped Biorthogonal Transforms Based on Coding Efficiency. IEEE International Conference on Image Processing, ICIP, Vol. 1. (1999) 624627.

11. Akkarakaran S. and Vaidyanathan P. P.: New Results and Open Problems on Nonuniform Filter-Banks. IEEE International Conference on Acoustics, Speech, and Signal Processing, ICASSP, Vol. 3. (1999) 1501-1504.

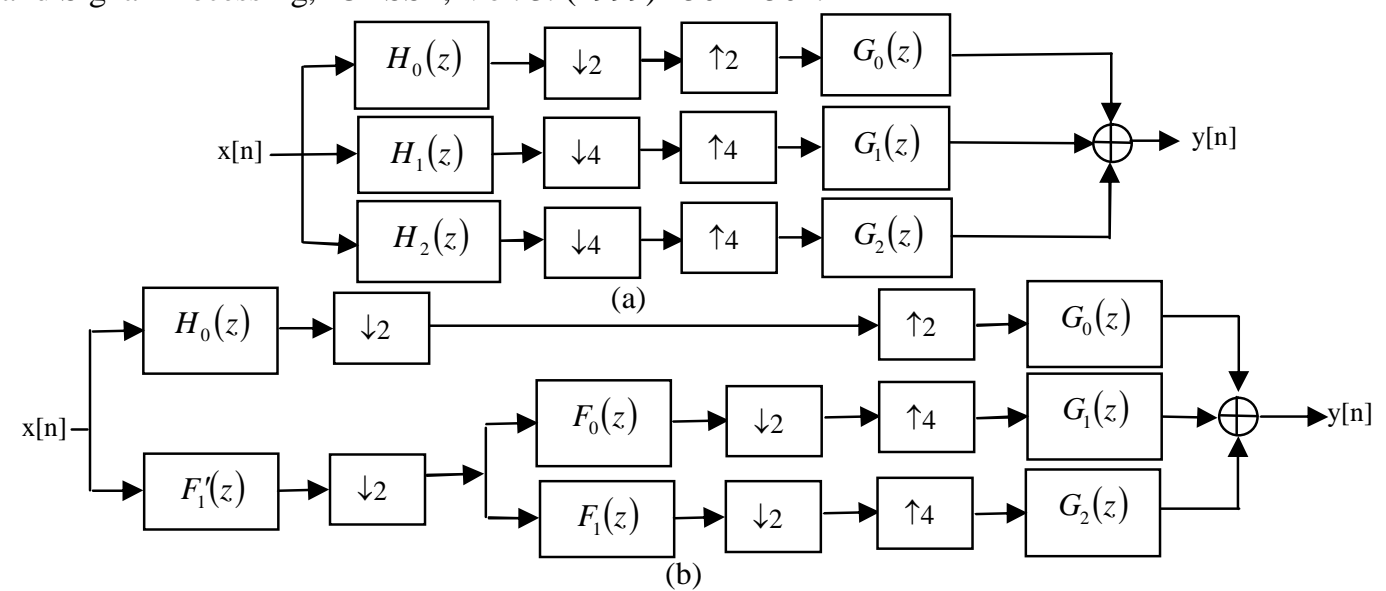

Fig. 1. (a) Non-uniform filter bank (b) Tree structure filter bank

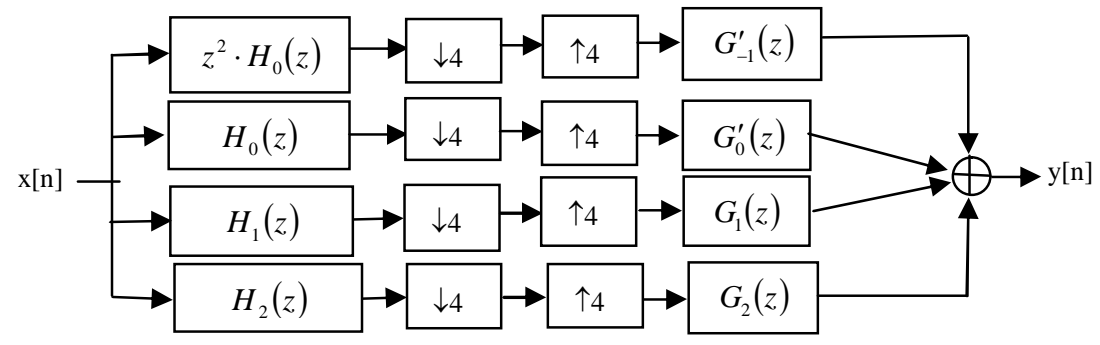

Fig. 2. Realization of non-uniform filter bank via a uniform filter bank 